\title{
The supermartingale property of the optimal wealth process for general semimartingales
}

\author{
Sara Biagini*and Marco Frittelli ${ }^{\dagger}$ \\ FINANCE AND STOCHASTICS ${ }^{\ddagger} 2007$ \\ Vol 11/2, pp. 253-266
}

\begin{abstract}
We consider a stochastic financial incomplete market where the price processes are described by a vector valued semimartingale that is possibly non locally bounded. We face the classical problem of the utility maximization from terminal wealth, under the assumption that the utility function is finite valued and smooth on the entire real line and satisfies Reasonable Asymptotic Elasticity. In this general setting, it was shown in Biagini and Frittelli [BF05] that the optimal claim admits an integral representation as soon as the minimax $\sigma-$ martingale measure is equivalent to the reference probability measure. We show that the optimal wealth process is in fact a supermartingale with respect to every $\sigma$-martingale measure with finite generalized entropy, thus extending the analogous result proved by Schachermayer [Sch03] in the locally bounded case.
\end{abstract}

Keywords: utility maximization, non locally bounded semimartingale, duality methods, optimal wealth process, $\sigma$-martingale measure.

JEL classification: G11, G12, G13 AMS classification: 60G42, 60G44

Running title: supermartingale property

\section{Introduction}

In this paper we complement the results in Biagini-Frittelli [BF05] by proving an additional important property of the optimal wealth process there studied. The comments on the literature are postponed to the end of this introduction.

We face the utility maximization problem

$$
\sup _{H \in \mathcal{H}} E\left[u\left(x+(H \cdot X)_{T}\right)\right]
$$

\footnotetext{
*Università degli Studi di Perugia, Italy. Email: sbiagini@unipg.it

${ }^{\dagger}$ Università degli Studi di Firenze, Italy. Email: Marco.Frittelli@dmd.unifi.it
}

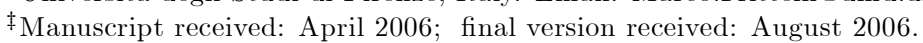


in which $u: \mathbb{R} \rightarrow \mathbb{R}$ is the utility function, $x \in \mathbb{R}$ is the constant initial endowment, $T \in(0, \infty]$ is a fixed time horizon, $X$ is an $\mathbb{R}^{d}$-valued càdlàg semimartingale defined on a filtered probability space $\left(\Omega, \mathcal{F},\left(\mathcal{F}_{t}\right)_{t \in[0, T]}, P\right)$ and $\mathcal{H}$ is the class of admissible integrands to be defined precisely later. In case $T=\infty$, we assume that, for every process $Y$ under consideration, the limit $Y_{\infty}=\lim _{t \uparrow+\infty} Y_{t}$ exists. The filtration satisfies the usual assumptions of right continuity and completeness and $\mathcal{F}_{0}$ is trivial, i.e. it is generated by the $P$-negligible sets in $\mathcal{F}_{T}$. The semimartingale $X$ models the discounted evolution of the prices of $d$ assets and it is not assumed to be locally bounded.

Assumption 1 The utility $u: \mathbb{R} \rightarrow \mathbb{R}$ is a strictly concave increasing differentiable function satisfying the Inada conditions

$$
\lim _{x \rightarrow-\infty} u^{\prime}(x)=\infty \text { and } \lim _{x \rightarrow \infty} u^{\prime}(x)=0,
$$

and having Reasonable Asymptotic Elasticity, as defined by Schachermayer [Sch01].

As in [BF05], we let $\Phi(y)=\sup _{x \in \mathbb{R}}\{u(x)-x y\}$ be the convex conjugate of the utility function $u$ and we set

$$
\begin{gathered}
\left.\mathcal{P}_{\Phi}=Q \ll P \mid E\left[\Phi\left(\frac{d Q}{d P}\right)\right]<\infty\right\}, \\
\mathcal{M}=\{Q \ll P \mid X \text { is a } \sigma-\text { martingale w.r.to } Q\} .
\end{gathered}
$$

Assumption $2 \mathcal{M} \cap \mathcal{P}_{\Phi} \neq \emptyset$.

We are now ready to introduce the class $\mathcal{H}$ of admissible integrands, i.e. the set of admissible trading strategies. In what follows, " $H \in L(X ; Q)$ " is a shorthand for " $H$ is predictable and $X$-integrable under the probability $Q$ ". When $Q$ is equivalent to $P$ we simply write $H \in L(X)$.

The classical way to define admissibility of an integrand $H \in L(X)$ is to require that the stochastic integral $H \cdot X$ is uniformly bounded from below by a constant. This concept was first introduced by Harrison and Pliska [HP81] and then applied by several authors when $X$ is locally bounded (see e.g. Schachermayer [Sch01] and the references therein).

In order to treat the general case, when $X$ can be non locally bounded, we considered in [BF05] the more general class

$$
\mathcal{H}^{W} \triangleq\left\{H \in L(X) \mid \exists c>0 \text { s.t. }(H \cdot X)_{t} \geq-c W, t \in[0, T]\right\}
$$

of integrands for which the stochastic integrals are bounded from below by a "sufficiently integrable" random variable $W \geq 1$. This natural extension of the notion of admissibility was already introduced in [Sch94] and [DS99], but it was only used in the context of the fundamental theorem of asset pricing. 
In [BF05] we required that $W$ satisfies two conditions that characterize the convex set $\mathcal{W}$ of loss variables. Similarly to [BF05], we will work under the following additional hypothesis.

Assumption $3 \mathcal{W}$ is not empty.

Remark 1 Assumption 3 is equivalent to the existence of processes $H^{i} \in L\left(X^{i}\right), i=$ $1, \ldots d$, which never hit zero (i.e. $P\left(\left\{\omega \mid \exists t>0\right.\right.$ s.t. $\left.\left.H_{t}^{i}(\omega)=0\right\}\right)=0$ ) and such that

$$
E\left[u\left(-c\left(1+\left(H^{i} \cdot X^{i}\right)_{T}^{*}\right)\right)\right]>-\infty \text { for all } c>0,
$$

where $^{*}$ stands for the maximal process.

Using this result, it is not difficult to see that $1 \in \mathcal{W}$ when $X$ is locally bounded, so Assumption 3 automatically holds. Hence the results hereafter presented are true extensions of those proven by Schachermayer [Sch03] in the locally bounded case.

On the other hand, it is clear that this assumption puts some restrictions on the jumps of $X$. For instance, let $u(x)=-e^{-x}$ and let $X$ be a scalar Compound Poisson process. Then, if the jump size is gaussian, $\mathcal{W} \neq \emptyset$; if the jump size has a doubly-exponential distribution (with probability density $f(x) \propto e^{-\lambda|x|}, \lambda>0$ ), $\mathcal{W}=\emptyset$.

We also showed that if $W \in \mathcal{W}$ then $W \in L^{1}(Q)$ for all $Q \in \mathcal{M} \cap \mathcal{P}_{\Phi}$. Henceforth, the bound in the definition (2) still permits to apply Ansel and Stricker result [AS94] and to deduce that if $H \in \mathcal{H}^{W}$ then

$$
H \cdot X \text { is a supermartingale under all } Q \in \mathcal{M} \cap \mathcal{P}_{\Phi}
$$

In economic terms the selection of the class $\mathcal{H}^{W}$ means that the losses admitted in the trading are pointwise bounded from below by some given $W$, which may be considered as an attractive feature for some economic agents. These agents face more risk in order to improve their maximum expected utility, i.e. they select $\mathcal{H}^{W}$ as domain in the utility maximization problem, thus solving the problem

$$
\sup _{H \in \mathcal{H}^{W}} E\left[u\left(x+(H \cdot X)_{T}\right)\right]
$$

As pointed out in [BF05], in the general framework of possibly non locally bounded semimartingales the supremum in (4) is not necessarily attained by a process $H \in \mathcal{H}^{W}$. However, the problem (4) can be reformulated in such a way that the optimal solution is a terminal value of a more general stochastic integral, not necessarily well-controlled by $W$.

Mathematically speaking, which is then the "good" class of integrands we should work with in order to catch the optimal solution? In Theorem 3 we prove that the appropriate domain is $\mathcal{H}$, i.e. the class of processes that verify condition (3).

Definition 2 The class $\mathcal{H}$ of admissible integrands is given by

$$
\mathcal{H}=\left\{H \in L(X) \mid H \cdot X \text { is a supermartingale under all } Q \in \mathcal{M} \cap \mathcal{P}_{\Phi}\right\}
$$


Without further mention, throughout the paper Assumptions 1, 2 and 3 are supposed to hold true. Hereafter there is the main result of the present paper.

Theorem 3 The following hold true.

I- For all $x \in \mathbb{R}$

$$
\sup _{H \in \mathcal{H}} E\left[u\left(x+(H \cdot X)_{T}\right)\right]=\min _{y>0, Q \in \mathcal{M} \cap \mathcal{P}_{\Phi}} y x+E\left[\Phi\left(y \frac{d Q}{d P}\right)\right]<u(\infty),
$$

and the minimum of the dual problem in (5) is attained by the couple $y(x) \in(0, \infty), Q_{y(x)} \in \mathcal{M} \cap \mathcal{P}_{\Phi}$.

II- If $Q_{y(x)}$ is equivalent to $P$ then there exists the optimal solution $H_{x} \in \mathcal{H}$ to the primal problem in (5), $H_{x} \cdot X$ is a uniformly integrable martingale under $Q_{y(x)}$ and

$$
u^{\prime}\left(x+\left(H_{x} \cdot X\right)_{T}\right)=y(x) \frac{d Q_{y(x)}}{d P} .
$$

In the particular case $u(x)=-e^{-\gamma x}, \gamma>0$, the optimal wealth process $H_{x} \cdot X$ is in fact a true martingale under each $Q \in \mathcal{M} \cap \mathcal{P}_{\Phi}$.

The literature on this topic The convex analysis approach to utility maximization from terminal wealth in semimartingale models was pioneered by Pliska [Pli86] and it has been successfully applied by many authors. Here we mention those articles that deal in particular with the martingale and supermartingale property of the optimal portfolio process in semimartingale models of financial markets.

This subject started with the Six Authors' paper [DGR+02]. Under the assumptions that $X$ is locally bounded, the utility is exponential and under an additional technical condition (the reverse Hölder inequality), it is proved in [DGR+02] that the optimal wealth process is a true martingale under every local martingale measure $Q$ with finite relative entropy. The technical condition was then shown to be superfluous by Kabanov and Stricker [KS02].

A third step was made by Schachermayer [Sch03]. For a general utility function $u: \mathbb{R} \rightarrow \mathbb{R}$ and for locally bounded $X$ he proved that if $Q_{y(x)} \sim P$, then the optimal solution is a supermartingale under every local martingale measure with finite generalized entropy. The "true martingale" property of the solution is in fact lost when $u$ is not exponential (see Proposition 4, [Sch03]).

Applying results from Theorem 1 [BF05], we will show that these properties still hold in the case of non locally bounded semimartingales. Part II in Theorem 3 is indeed the essential contribution of this paper. In our proof we closely follow the original and tricky Schachermayer's approach. However, the extension is non trivial, since we do not work with the set of local martingale measures (which is closed in $L^{1}(P)$ when $X$ is locally bounded), but with the 
set $\mathcal{M}$ of $\sigma$-martingale measures, which is not necessarily $L^{1}$-closed. Hence, the dynamic dual problem introduced in [Sch03] in our case does not necessarily have a minimum.

In the next section we prove those statements of Theorem 3 that can be immediately deduced from the results in [BF05]. In Items (a) and (b) below we isolate the key steps in the proof of Theorem 3 and in Section 4 we conclude it.

\section{First part of the proof of Theorem 3}

Note that each $H \in \mathcal{H}^{W}$ satisfies the property (3). This shows that $\mathcal{H}^{W} \subseteq \mathcal{H}$. From the definition of $\Phi$ we get $u\left(x+(H \cdot X)_{T}\right) \leq\left(x+(H \cdot X)_{T}\right) y \frac{d Q}{d P}+\Phi\left(y \frac{d Q}{d P}\right)$ for all $y>0$ and $Q \in \mathcal{M} \cap \mathcal{P}_{\Phi}$, so that $\operatorname{LHS}(5) \leq R H S(5)$.

The latter inequality and $\mathcal{H}^{W} \subseteq \mathcal{H}$ guarantee that

$$
\sup _{H \in \mathcal{H}^{W}} E\left[u\left(x_{0}+(H \cdot X)_{T}\right)\right]<u(\infty)
$$

for some $x_{0} \in \mathbb{R}$ and therefore the assumption stated in equation (12) in [BF05] is satisfied. Assumption 1 implies the assumptions (1)+(2) in [BF05], so we may apply Theorem 1 [BF05] and conclude that

(i) for all $x \in \mathbb{R}$

$$
\begin{aligned}
\sup _{H \in \mathcal{H}^{W}} E\left[u\left(x+(H \cdot X)_{T}\right)\right] & =\sup _{H \in \mathcal{H}} E\left[u\left(x+(H \cdot X)_{T}\right)\right] \\
& =\min _{y>0, Q \in \mathcal{M} \cap \mathcal{P}_{\Phi}} y x+E\left[\Phi\left(y \frac{d Q}{d P}\right)\right]
\end{aligned}
$$

and the minimum of the dual problem in (7) is attained by a unique couple $y(x) \in(0, \infty), Q_{y(x)} \in \mathcal{M} \cap \mathcal{P}_{\Phi}$.

(ii) if $Q_{y(x)}$ is equivalent to $P$ then there exists $H_{x} \in L(X)$ such that

$$
E\left[u\left(x+\left(H_{x} \cdot X\right)_{T}\right)\right]=\min _{y>0, Q \in \mathcal{M} \cap \mathcal{P}_{\Phi}} y x+E\left[\Phi\left(y \frac{d Q}{d P}\right)\right],
$$

$H_{x} \cdot X$ is a uniformly integrable martingale under $Q_{y(x)}$ and (6) holds true.

Comparing (i) and (ii) with (I) and (II) of Theorem 3, we see that what is left to show is only that, when $Q_{y(x)} \sim P$,

(a) $H_{x} \cdot X$ is a supermartingale under each $Q \in \mathcal{M} \cap \mathcal{P}_{\Phi}$;

(b) $H_{x} \cdot X$ is a true martingale under each $Q \in \mathcal{M} \cap \mathcal{P}_{\Phi}$, in case of the exponential utility.

The proofs of these two items will be carried on in Section 4. We will see that Item (b) follows easily from the results in [KS02]. To prove Item (a) we consider a dynamic problem, similar to that in [Sch03] but suited to our context. 


\section{An auxiliary dynamic problem}

From now on, $Q_{y(x)} \sim P$. In this section $\tau$ is any stopping time with values in $[0, T]$. Let $\left({ }^{\tau} X\right)_{t} \triangleq X_{t}-X_{\tau \wedge t}, t \geq 0$, be the increment of the process $X$ after $\tau$ and set

$$
\mathcal{M}(\tau) \triangleq\left\{Q \ll P \mid{ }^{\tau} X \text { is a } \sigma \text {-martingale under } Q\right\} .
$$

It is easily seen that, given a couple of stopping times $\tau_{1} \leq \tau_{2}, \mathcal{M}\left(\tau_{1}\right)$ is contained in $\mathcal{M}\left(\tau_{2}\right)$. This in particular implies that $\mathcal{M}=\mathcal{M}(0)$ is always a (convex) subset of $\mathcal{M}(\tau)$. Define also

$$
\mathcal{M}_{1}(\tau) \triangleq\left\{Q \in \mathcal{M}(\tau) \mid E^{\tau}\left[\frac{d Q}{d P}\right]=1\right\}
$$

where $E^{\tau}[\cdot]=E\left[\cdot \mid \mathcal{F}_{\tau}\right]$. Since $\mathcal{F}_{0}$ is trivial, when $\tau=0$ we get $\mathcal{M}=\mathcal{M}(0)=$ $\mathcal{M}_{1}(0)$.

Notations We denote with $Z_{t}=E^{t}\left[Z_{T}\right]$ the martingale process associated to the generic $Z_{T} \in L^{1}\left(\Omega, \mathcal{F}_{T}, P\right)$.

When $\mathcal{N}$ is a set of probabilities absolutely continuous w.r.t. $P, \mathcal{N}^{e}$ indicates the subset of the equivalent ones.

If $Z_{T}=\frac{d Q}{d P}$ and $Q \in \mathcal{N}\left(Q \in \mathcal{N}^{e}\right)$, we write: $Z_{T} \in \mathcal{N}\left(Z_{T} \in \mathcal{N}^{e}\right.$ respectively), thus identifying as usual the measure $Q \ll P$ with its density wrt $P$.

If $Q \in \mathcal{M}_{1}(\tau)$, we typically use the notation $Y$ instead of $Z$ for the density process of $Q$.

Lemma 4 For all $\tau, \mathcal{M}_{1}(\tau)$ is not empty.

Proof. The optimal density $\frac{d Q_{y(x)}}{d P} \triangleq \widehat{Z}_{T} \in \mathcal{M}^{e}$, so that $\widehat{Z}_{\tau}>0$ and $\widehat{Y}_{T} \triangleq \frac{\widehat{Z}_{T}}{\widehat{Z}_{\tau}} \in \mathcal{M}_{1}(\tau)$.

Lemma 5 Given a process $S$ and two $\sigma$-martingale probabilities for $S$, say $Q_{1}, Q_{2}$, then there exists a process $\phi \in L\left(S ; Q_{1}\right) \cap L\left(S ; Q_{2}\right)$, which is strictly positive under $P$, such that the process $\phi \cdot S$ is a martingale under both $Q_{1}, Q_{2}$.

Proof. This claim is not completely obvious, since $Q_{1}$ and $Q_{2}$ are not necessarily equivalent to $P$. By the definition of a $\sigma$-martingale (as given in [DS98]), for $i=1,2$ there exists a process $\varphi_{i} \in L\left(S ; Q_{i}\right)$, which is $Q_{i}$-strictly positive, such that $\varphi_{i} \cdot S$ is a $Q_{i}$-martingale. As it is well known, we can even suppose that $\varphi_{i} \cdot S$ is an $H^{1}\left(Q_{i}\right)$-martingale. Define $A_{i}=\left\{(\omega, t) \mid \varphi_{i}(t, \omega) \leq 0\right\}$. Then $A_{i}$ is predictable and $Q_{i}\left(A_{i}\right)=0$. Consider now $\widetilde{\varphi}_{i}=\varphi_{i} I_{A_{i}^{c}}+I_{A_{i}}$. Clearly, $\widetilde{\varphi_{i}}>0$ under $P$ and $\widetilde{\varphi_{i}} \cdot S=\varphi_{i} \cdot S$ under $Q_{i}$. Take $\phi=\widetilde{\varphi_{1}} \wedge \widetilde{\varphi_{2}}$ and note that it belongs to $L\left(S ; Q_{1}\right) \cap L\left(S ; Q_{2}\right)$, it is strictly positive under $P$ and $\phi \cdot S$ is an $H^{1}$-martingale with respect to both measures $Q_{1}, Q_{2}$.

The following Lemmata list some useful properties of $\mathcal{M}_{1}(\tau)$. By the previous Lemma we can work as if ${ }^{\tau} X$ were a true martingale under the measures $Q_{1}, Q_{2}$ considered (by passing to $\phi \cdot{ }^{\tau} X$ ). Taking this into account, the proofs become classical and we omit them (for more details, see e.g. [Del06]). 
Lemma 6 (Random convexity) Fix $Y_{T}^{1}, Y_{T}^{2} \in \mathcal{M}_{1}(\tau)$ and two nonnegative $\mathcal{F}_{\tau^{-}}$ measurable random variables $\eta_{1}, \eta_{2}$ such that $\eta_{1}+\eta_{2}=1$. Then $Y_{T}=\eta_{1} Y_{T}^{1}+$ $\eta_{2} Y_{T}^{2} \in \mathcal{M}_{1}(\tau)$. Of course, if $Y_{T}^{i} \in \mathcal{M}_{1}^{e}(\tau)$ then $Y_{T}$ also is in $\mathcal{M}_{1}^{e}(\tau)$.

Lemma 7 (Stability by concatenation) Let $Y_{T} \in \mathcal{M}_{1}(\tau)$.

If $\eta \geq 0$ is $\mathcal{F}_{\tau}$-measurable, with $E[\eta]=1$, then $Z_{T}=\eta Y_{T} \in \mathcal{M}(\tau)$.

If $\eta=Z_{\tau}$, where $Z_{T} \in \mathcal{M}$, then $\widetilde{Z_{T}}=Z_{\tau} Y_{T}$ is also in $\mathcal{M}$.

The next Lemma will be used in the proof of Proposition 12 and it is a well known consequence of the Reasonable Asymptotic Elasticity of $u$ (see Corollary 4.2 Schachermayer [Sch01]).

Lemma 8 These two conditions hold true:

- For each compact interval $\left[\lambda_{0}, \lambda_{1}\right]$ contained in $(0, \infty)$ there exist constants $\alpha>0$ and $\beta>0$ such that

$$
\Phi(\lambda y) \leq \alpha \Phi(y)+\beta(y+1), \text { for } y>0 \text { and } \lambda \in\left[\lambda_{0}, \lambda_{1}\right] .
$$

- There exist constants $\gamma>0$ and $\delta>0$ such that

$$
y\left|\Phi^{\prime}(y)\right| \leq \gamma \Phi(y)+\delta(y+1), \text { for } y>0 .
$$

As a consequence of (8)

$$
E\left[\Phi\left(\lambda Z_{T}\right)\right]<\infty \text { for all } \lambda>0 \text { and } Z_{T} \in \mathcal{P}_{\Phi} .
$$

For any fixed, positive $\mathcal{F}_{\tau}$-measurable random variable $\zeta$, we finally define the dynamic minimization problem

$$
v_{\tau}(\zeta) \triangleq \operatorname{ess}_{\inf _{Y_{T} \in \mathcal{M}_{1}(\tau)} E^{\tau}\left[\Phi\left(\zeta Y_{T}\right)\right]}
$$

This $v_{\tau}$ is always well defined, since $\Phi$ is bounded from below, but it evidently has a quite complex functional dependence on $\zeta$.

Remark 9 Note that when $\tau=0$, problem (11) reduces to the static minimization

$$
v_{0}(y)=\inf _{Z_{T} \in \mathcal{M}} E\left[\Phi\left(y Z_{T}\right)\right], \quad y>0 .
$$

From Proposition 3 [BF05] we know that, for all $y>0$, the inf in (12) is reached. On the contrary, in (11) the ess inf may not be attained. However, we will see that the ess inf is attained in the case of interest (Corollary 14).

In the sequel we will always assume that $v_{\tau}$ is defined over the domain

$$
\mathcal{V}_{\tau}=\left\{\zeta \in L^{0}\left(\Omega, \mathcal{F}_{\tau}, P\right) \mid \zeta>0 \text { and } E\left[\Phi\left(\zeta Y_{T}\right)\right]<\infty \text { for some } Y_{T} \in \mathcal{M}_{1}(\tau)\right\} \text {, }
$$

so that $v_{\tau}(\zeta)$ will be finite-valued and $P$-integrable. 
Remark 10 By convexity of $\Phi$ and by condition (8) above, it is easy to see that the domain $\mathcal{V}_{\tau}$ is a convex cone (but not containing the origin).

The following Lemma provides a variety of elements of $\mathcal{V}_{\tau}$.

Lemma 11 If $Z_{T} \in \mathcal{M}^{e} \cap \mathcal{P}_{\Phi}$, then $y Z_{\tau} \in \mathcal{V}_{\tau}$ for any positive constant $y$.

Proof. Set $\zeta=Z_{\tau}$ and define $Y_{T} \triangleq \frac{Z_{T}}{Z_{\tau}}$. Then $Y_{T} \in \mathcal{M}_{1}(\tau)$ and $E\left[\Phi\left(\zeta Y_{T}\right)\right]=$ $E\left[\Phi\left(Z_{T}\right)\right]<\infty$. This means $Z_{\tau} \in \mathcal{V}_{\tau}$ and by the cone property of $\mathcal{V}_{\tau}$ we conclude the proof.

Proposition 12 Let $v_{\tau}$ be defined over $\mathcal{V}_{\tau}$.

1. $v_{\tau}(\zeta)$ is a bounded from below, $P$-integrable $\mathcal{F}_{\tau}$-measurable random variable.

The set of $\mathcal{F}_{\tau}$-measurable random variables

$$
\left\{E^{\tau}\left[\Phi\left(\zeta Y_{T}\right)\right] \mid Y_{T} \in \mathcal{M}_{1}(\tau)\right\}
$$

is directed downward, so that there exists a minimizing sequence $\left(Y_{T}^{n}\right)_{n}$ in $\mathcal{M}_{1}(\tau)$ satisfying

$$
E^{\tau}\left[\Phi\left(\zeta Y_{T}^{n}\right)\right] \downarrow v_{\tau}(\zeta) \quad P \text {-a.s. and also in } L^{1}(P) .
$$

2. (Random convexity of $v_{\tau}$ ) For any $A \in \mathcal{F}_{\tau}$, any $\mathcal{F}_{\tau}$-measurable random variables $\alpha$ and $\beta$ satisfying $\alpha 1_{A} \geq 0, \beta 1_{A} \geq 0,(\alpha+\beta) 1_{A}=1_{A}$, and any $\zeta_{i}, i=1,2,3$, we have

$$
v_{\tau}\left(\left(\alpha \zeta_{1}+\beta \zeta_{2}\right) 1_{A}+\zeta_{3} 1_{A^{c}}\right) 1_{A} \leq\left(\alpha v_{\tau}\left(\zeta_{1}\right)+\beta v_{\tau}\left(\zeta_{2}\right)\right) 1_{A} .
$$

3. (On $\left.v_{\tau}^{\prime}\right)$ The right derivative $v_{\tau}^{\prime}(\zeta) \triangleq \lim _{\lambda \downarrow 1} \frac{v_{\tau}(\lambda \zeta)-v_{\tau}(\zeta)}{\lambda \zeta-\zeta}$ is well defined and it verifies

$$
\zeta v_{\tau}^{\prime}(\zeta) \leq C_{1} v_{\tau}(\zeta)+C_{2}(\zeta+1), \quad \text { for some } C_{i}>0 .
$$

Moreover, if the essential infimum in $v_{\tau}(\zeta)$ is reached by $Y_{T}^{\zeta}$, i.e.

$$
v_{\tau}(\zeta)=E^{\tau}\left[\Phi\left(\zeta Y_{T}^{\zeta}\right)\right]
$$

then $v_{\tau}^{\prime}$ can be explicitly expressed as

$$
E^{\tau}\left[\Phi^{\prime}\left(\zeta Y_{T}^{\zeta}\right) Y_{T}^{\zeta}\right]=v_{\tau}^{\prime}(\zeta)
$$

4. (Monotonicity of $v_{\tau}^{\prime}$ ) If $\zeta_{2} \geq \lambda_{0} \zeta_{1}$ for some constant $\lambda_{0}>1$ on a set $A \in \mathcal{F}_{\tau}$, then

$$
v_{\tau}^{\prime}\left(\zeta_{2}\right) 1_{A} \geq v_{\tau}^{\prime}\left(\zeta_{1}\right) 1_{A}
$$


Proof. 1. We have already seen that $v_{\tau}(\zeta)$ is bounded from below and integrable. Now we prove that the set in (14) is directed downward. Let $Y_{T}^{i} \in$ $\mathcal{M}_{1}(\tau)$ and set $v^{i} \triangleq E^{\tau}\left[\Phi\left(\zeta Y_{T}^{i}\right)\right]$. Then $v^{1} \wedge v^{2}$ coincides with

$$
E^{\tau}\left[\Phi\left(\zeta Y_{T}\right)\right], \quad Y_{T}=1_{\left\{v^{1} \leq v^{2}\right\}} Y_{T}^{1}+1_{\left\{v^{2}<v^{1}\right\}} Y_{T}^{2}
$$

and $Y_{T} \in \mathcal{M}_{1}(\tau)$ by Lemma 6 . Hence, by the definition of the essential infimum there exists a sequence $\left(Y_{T}^{n}\right)_{n}$ in $\mathcal{M}_{1}(\tau)$ such that $v_{n} \triangleq E^{\tau}\left[\Phi\left(\zeta Y_{T}^{n}\right)\right] \downarrow v_{\tau}(\zeta)$ $P$-a.s. To get a sequence which converges also in $L^{1}(P)$, fix an $\bar{Y}_{T}$ satisfying the condition in (13), set $\bar{v} \triangleq E^{\tau}\left[\Phi\left(\zeta \bar{Y}_{T}\right)\right]$ and consider

$$
\tilde{Y}_{T}^{n}=1_{\left\{\bar{v} \leq v^{n}\right\}} \bar{Y}_{T}+1_{\left\{v^{n}<\bar{v}\right\}} Y_{T}^{n}
$$

Then

$$
v_{\tau}(\zeta) \leq w^{n} \triangleq E^{\tau}\left[\Phi\left(\zeta \widetilde{Y}_{T}^{n}\right)\right]=v^{n} \wedge \bar{v} \leq \bar{v} \text { and } E[\bar{v}]=E\left[\Phi\left(\zeta \bar{Y}_{T}\right)\right]<\infty
$$

so that $w^{n} \downarrow v_{\tau}(\zeta) P$-a.s. and also in $L^{1}(P)$ by dominated convergence.

2. Define $\widetilde{\zeta}=I_{A}\left(\alpha \zeta_{1}+\beta \zeta_{2}\right)+I_{A^{c}} \zeta_{3}$. By convexity of $\Phi$ and $\mathcal{F}_{\tau^{-}}$-measurability of $\alpha, \beta, I_{A}$ we get, for all $Y_{T}^{1}, Y_{T}^{2}, Y_{T}^{3} \in \mathcal{M}_{1}(\tau)$

$$
\begin{aligned}
& I_{A}\left(\alpha E^{\tau}\left[\Phi\left(\zeta_{1} Y_{T}^{1}\right)\right]+\beta E^{\tau}\left[\Phi\left(\zeta_{2} Y_{T}^{2}\right)\right]\right) \\
\geq & E^{\tau}\left[I_{A} \Phi\left(\alpha \zeta_{1} Y_{T}^{1}+\beta \zeta_{2} Y_{T}^{2}\right)\right] \\
= & E^{\tau}\left[I_{A} \Phi\left(\widetilde{\zeta} Y_{T}\right)\right] \geq I_{A} v_{\tau}(\widetilde{\zeta})
\end{aligned}
$$

where $Y_{T}=I_{A}\left(\frac{\alpha \zeta_{1}}{\alpha \zeta_{1}+\beta \zeta_{2}} Y_{T}^{1}+\frac{\beta \zeta_{2}}{\alpha \zeta_{1}+\beta \zeta_{2}} Y_{T}^{2}\right)+I_{A^{c}} Y_{T}^{3} \in \mathcal{M}_{1}(\tau)$ by Lemma 6. Now, just take the essential infimum over $Y_{T}^{1}, Y_{T}^{2}$ and conclude.

3. Consider the function $v_{\tau, \zeta}:(0, \infty) \rightarrow L^{1}\left(\Omega, \mathcal{F}_{\tau}, P\right)$ defined by $v_{\tau, \zeta}(\lambda) \triangleq$ $v_{\tau}(\lambda \zeta)$. This is evidently a convex function of $\lambda$ so the right derivative $D^{+} v_{\tau, \zeta}(\lambda)$ is well defined and

$$
D^{+} v_{\tau, \zeta}(1) \triangleq \lim _{\lambda \downarrow 1} \frac{v_{\tau, \zeta}(\lambda)-v_{\tau, \zeta}(1)}{\lambda-1}=\zeta v_{\tau}^{\prime}(\zeta)
$$

Let $Y_{T}^{n}(\lambda) \in \mathcal{M}_{1}(\tau)$ (resp. $\left.W_{T}^{n} \in \mathcal{M}_{1}(\tau)\right)$ be a minimizing sequence for $v_{\tau}(\lambda \zeta)$ (resp. $v_{\tau}(\zeta)$ ) as in item 1) above. We therefore have

$$
D^{+} v_{\tau, \zeta}(1)=\lim _{\lambda \downarrow 1} \lim _{n \uparrow \infty} \frac{E^{\tau}\left[\Phi\left(\lambda \zeta Y_{T}^{n}(\lambda)\right)-\Phi\left(\zeta W_{T}^{n}\right)\right]}{\lambda-1}
$$

We can always suppose that

$$
E^{\tau}\left[\Phi\left(\lambda \zeta Y_{T}^{n}(\lambda)\right)\right] \leq E^{\tau}\left[\Phi\left(\lambda \zeta W_{T}^{n}\right)\right]
$$

if not, just use random convexity as in the proof of item 1) to replace $Y_{T}^{n}(\lambda)$ with a suitable one. Hence

$$
D^{+} v_{\tau, \zeta}(1) \leq \lim _{\lambda \downarrow 1} \liminf _{n \uparrow \infty} \frac{E^{\tau}\left[\Phi\left(\lambda \zeta W_{T}^{n}\right)-\Phi\left(\zeta W_{T}^{n}\right)\right]}{\lambda-1}
$$


and, by convexity of $\Phi$,

$$
D^{+} v_{\tau, \zeta}(1) \leq \lim _{\lambda \downarrow 1} \liminf _{n \uparrow \infty} E^{\tau}\left[\Phi^{\prime}\left(\lambda \zeta W_{T}^{n}\right) \zeta W_{T}^{n}\right] .
$$

Applying first (9) and then (8) we get

$$
\begin{aligned}
& E^{\tau}\left[\Phi^{\prime}\left(\lambda \zeta W_{T}^{n}\right) \zeta W_{T}^{n}\right] \\
\leq & \frac{1}{\lambda}\left\{\gamma E^{\tau}\left[\Phi\left(\lambda \zeta W_{T}^{n}\right)\right]+\delta(\lambda \zeta+1)\right\} \\
\leq & \frac{\gamma}{\lambda}\left\{E^{\tau}\left[\alpha \Phi\left(\zeta W_{T}^{n}\right)\right]+\beta(\zeta+1)\right\}+\delta\left(\zeta+\frac{1}{\lambda}\right) \\
\leq & C_{1} E^{\tau}\left[\Phi\left(\zeta W_{T}^{n}\right)\right]+C_{2}(\zeta+1)
\end{aligned}
$$

where the last inequality holds for all $\lambda \geq 1$. Therefore

$$
D^{+} v_{\tau, \zeta}(1) \leq \liminf _{n} C_{1} E^{\tau}\left[\Phi\left(\zeta W_{T}^{n}\right)\right]+C_{2}(\zeta+1)=C_{1} v_{\tau}(\zeta)+C_{2}(\zeta+1)
$$

and (15) follows from (19).

Suppose now that the ess inf is reached by $Y_{T}^{\zeta}$ and consider the auxiliary function $h:(0, \infty) \rightarrow L^{1}\left(\Omega, \mathcal{F}_{\tau}, P\right)$ defined as

$$
h(\lambda)=E^{\tau}\left[\Phi\left(\lambda \zeta Y_{T}^{\zeta}\right)\right]
$$

Then $h$ is bounded from below and convex, $h(\lambda) \geq v_{\tau, \zeta}(\lambda) \triangleq v_{\tau}(\lambda \zeta)$ and, from $(16), h(1)=v_{\tau, \zeta}(1)$. Hence

$$
D^{-} h(1) \leq D^{-} v_{\tau, \zeta}(1) \leq D^{+} v_{\tau, \zeta}(1) \leq D^{+} h(1)
$$

In addition, $h$ is clearly differentiable by condition (9). The expression for the derivative is

$$
\left.\frac{d}{d \lambda} h(\lambda)\right|_{\lambda=1}=E^{\tau}\left[\Phi^{\prime}\left(\zeta Y_{T}^{\zeta}\right) \zeta Y_{T}^{\zeta}\right]
$$

So equalities must hold in (20) and we derive (17).

4. As shown below, the convexity of $v_{\tau}(\cdot)$ implies that

$$
\frac{v_{\tau}\left(\zeta_{3}\right)-v_{\tau}\left(\zeta_{1}\right)}{\zeta_{3}-\zeta_{1}} 1_{A} \leq \frac{v_{\tau}\left(\zeta_{4}\right)-v_{\tau}\left(\zeta_{2}\right)}{\zeta_{4}-\zeta_{2}} 1_{A}
$$

for any $\zeta_{i}$ satisfying on the set $A$ the inequalities $0<\zeta_{1}<\zeta_{3}<\zeta_{2}<\zeta_{4}$. Since on the set $A$ in question we have $0<\zeta_{1}<\lambda \zeta_{1}<\zeta_{2}<\delta \zeta_{2}$ for all $\lambda \in\left(1, \lambda_{0}\right)$ and for all $\delta>1$, we get

$$
\begin{aligned}
1_{A} v_{\tau}^{\prime}\left(\zeta_{1}\right) & =\lim _{\lambda \downarrow 1} 1_{A} \frac{v_{\tau}\left(\lambda \zeta_{1}\right)-v_{\tau}\left(\zeta_{1}\right)}{\lambda \zeta_{1}-\zeta_{1}} \\
& \leq \lim _{\delta \downarrow 1} 1_{A} \frac{v_{\tau}\left(\delta \zeta_{2}\right)-v_{\tau}\left(\zeta_{2}\right)}{\delta \zeta_{2}-\zeta_{2}}=1_{A} v_{\tau}^{\prime}\left(\zeta_{2}\right)
\end{aligned}
$$


To check (21) first suppose that $0<\zeta_{1}<\zeta_{3}<\zeta_{2}$ holds on $A$ and define the $\mathcal{F}_{\tau}$-measurable random variables $\alpha=\frac{\zeta_{2}-\zeta_{3}}{\zeta_{2}-\zeta_{1}}$ and $\beta=\frac{\zeta_{3}-\zeta_{1}}{\zeta_{2}-\zeta_{1}}$. Then $\alpha 1_{A} \geq 0$, $\beta 1_{A} \geq 0,(\alpha+\beta) 1_{A}=1_{A}$ and $\left(\alpha \zeta_{1}+\beta \zeta_{2}\right) 1_{A}=\zeta_{3} 1_{A}$. From item 2) we get

$$
\begin{gathered}
v_{\tau}\left(\zeta_{3}\right) 1_{A}=v_{\tau}\left(\zeta_{3} 1_{A}+\zeta_{3} 1_{A^{c}}\right) 1_{A} \leq\left[\alpha v_{\tau}\left(\zeta_{1}\right)+\beta v_{\tau}\left(\zeta_{2}\right)\right] 1_{A}, \\
{\left[v_{\tau}\left(\zeta_{3}\right)-v_{\tau}\left(\zeta_{1}\right)\right] 1_{A} \leq\left[(\alpha-1) v_{\tau}\left(\zeta_{1}\right)+\beta v_{\tau}\left(\zeta_{2}\right)\right] 1_{A}} \\
=\beta\left[v_{\tau}\left(\zeta_{2}\right)-v_{\tau}\left(\zeta_{1}\right)\right] 1_{A} \leq \frac{\beta}{\alpha}\left[v_{\tau}\left(\zeta_{2}\right)-v_{\tau}\left(\zeta_{3}\right)\right] 1_{A} .
\end{gathered}
$$

Taking $0<\zeta_{3}<\zeta_{2}<\zeta_{4}$, and computing similarly $v_{\tau}\left(\zeta_{2}\right) 1_{A}$, one easily deduces (21).

Remark 13 In item 4) it is required not only that $\zeta_{2}$ is greater than $\zeta_{1}$ but also that $\frac{\zeta_{2}}{\zeta 1}$ is uniformly strictly bigger than 1 . So only a weak form of monotonicity for $v_{\tau}^{\prime}$ is shown. Nevertheless, to prove (18) it is sufficient that the inequality $\frac{\zeta_{2}}{\zeta 1} \geq \lambda_{0}>1$ holds true only over the set $A$. This result will be used in Section 4 in the proof of Item (a).

Corollary 14 Let $y$ be such that the minimal $\widehat{Z}_{T}(y)$ solution of problem (12) is equivalent to $P$, i.e. $\widehat{Z}_{T}(y) \in \mathcal{M}^{e} \cap \mathcal{P}_{\Phi}$. Then

$$
v_{\tau}\left(y \widehat{Z}_{\tau}(y)\right)=E^{\tau}\left[\Phi\left(y \widehat{Z}_{T}(y)\right)\right]
$$

i.e. the optimal solution to $v_{\tau}\left(y \widehat{Z}_{\tau}(y)\right)$ exists and coincides with $\widehat{Y}_{T}=\frac{\widehat{Z}_{T}(y)}{\widehat{Z}_{\tau}(y)} \in$ $\mathcal{M}_{1}(\tau)$. So, from the expression of $v_{\tau}^{\prime}$ in Proposition 12,

$$
E^{\tau}\left[\Phi^{\prime}\left(y \widehat{Z}_{T}(y)\right) \frac{\widehat{Z}_{T}(y)}{\widehat{Z}_{\tau}(y)}\right]=v_{\tau}^{\prime}\left(y \widehat{Z}_{\tau}(y)\right)
$$

Proof. Set $\zeta=y \widehat{Z}_{\tau}(y)$. Since $y \widehat{Z}_{T}(y)=\zeta \widehat{Y}_{T}, v_{\tau}(\zeta) \leq E^{\tau}\left[\Phi\left(y \widehat{Z}_{T}(y)\right)\right]$ is obvious by definition of $v_{\tau}$. To show that the equality must hold, suppose that

$$
P\left(v_{\tau}(\zeta)<E^{\tau}\left[\Phi\left(y \widehat{Z}_{T}(y)\right)\right]\right)>0,
$$

From item 1) in Proposition 12, we can select a sequence $Y_{T}^{n} \in \mathcal{M}_{1}(\tau)$ such that $E^{\tau}\left[\Phi\left(\zeta Y_{T}^{n}\right)\right] \downarrow v_{\tau}\left(y \widehat{Z}_{\tau}(y)\right)$ in $L^{1}(P)$. Set $Z_{T}^{n} \triangleq \widehat{Z}_{\tau}(y) Y_{T}^{n}$. By Lemma $7, Z_{T}^{n}$ belongs to $\mathcal{M}$. Note also

$$
E\left[\Phi\left(y Z_{T}^{n}\right)\right]=E\left[\Phi\left(\zeta Y_{T}^{n}\right)\right] \downarrow E\left[v_{\tau}\left(y \widehat{Z}_{\tau}(y)\right)\right]<E\left[\Phi\left(y \widehat{Z}_{T}(y)\right)\right]
$$

and this is a contradiction with the minimality of $\widehat{Z}_{T}(y)$ in the problem (12). 


\section{Conclusion of the proof of Theorem 3}

We are ready to prove Items (a) and (b) stated in Section 2. Though closely following [Sch03], we emphasize that we collected the results needed in our generalized proof in Proposition 12 and Corollary 14.

First of all, we rewrite Lemma 1 in [Sch03] (see also the other references there cited) in $\sigma$-martingale terms.

Lemma 15 Let $X=\left(X_{t}\right)_{0 \leq t \leq T}$ be a $\sigma$-martingale under $Q, H \in L(X ; Q)$ and $S=H \cdot X$. If, for any sequence of stopping times $\left(\tau_{n}\right)_{n=1}^{\infty}$ with values in $[0, T] \cup\{\infty\}$ and increasing to $\infty$, we have

$$
\lim _{n \rightarrow \infty} \sup E_{Q}\left[S_{\tau_{n}} I_{\left\{\tau_{n}<\infty, S_{\tau_{n}} \leq 0\right\}}\right]=0
$$

then $S$ is a supermartingale under $Q$.

\subsection{Proof of Item (a)}

To simplify the notations we set $\widehat{Q}=Q_{y(x)} \sim P$ and $y=y(x)$. By contradiction we suppose there exists a $Q \in \mathcal{M} \cap \mathcal{P}_{\Phi}$ such that $S=x+H_{x} \cdot X$ is not a $Q$ supermartingale. We may assume that $Q \sim P$, otherwise we may replace $Q$ with $\frac{Q+\widehat{Q}}{2}$, since this latter probability belongs to $\mathcal{M}^{e} \cap \mathcal{P}_{\Phi}$ and doesn't turn $S$ into a supermartingale. We set $Z_{T}=\frac{d Q}{d P}$ and $\widehat{Z}_{T}=\frac{d \widehat{Q}}{d P}$. Then, from Lemma 15 there exists a sequence of stopping times $\left(\tau_{n}\right)_{n=1}^{\infty}$, increasing to $\infty$, such that

$$
\liminf _{n} E\left[-Z_{\tau_{n}} S_{\tau_{n}} I_{\left\{\tau_{n}<\infty, S_{\tau_{n}} \leq 0\right\}}\right]>0
$$

Since $S$ is a uniformly integrable martingale under $\widehat{Q}$ (see item (ii), Section 2), we also know that

$$
\lim _{n \rightarrow \infty} E\left[\widehat{Z}_{\tau_{n}} S_{\tau_{n}} I_{\left\{\tau_{n}<\infty, S_{\tau_{n}} \leq 0\right\}}\right]=0
$$

Fix $\lambda_{0}>1$. From (23) it follows

$$
\begin{aligned}
& \left.\liminf _{n} E\left[-Z_{\tau_{n}} S_{\tau_{n}} I_{\left\{\tau_{n}<\infty, S_{\tau_{n}} \leq 0\right\}} I_{\left\{Z_{\tau_{n}}>\lambda_{0}\right.} \widehat{Z}_{\tau_{n}}\right\}\right]+ \\
& \liminf _{n} E\left[-Z_{\tau_{n}} S_{\tau_{n}} I_{\left\{\tau_{n}<\infty, S_{\tau_{n}} \leq 0\right\}} I_{\left\{Z_{\tau_{n}} \leq \lambda_{0} \widehat{Z}_{\tau_{n}}\right\}}\right]>0
\end{aligned}
$$

The second term in (24) is equal to zero because

$$
\begin{aligned}
0 & \geq \liminf _{n} E\left[Z_{\tau_{n}} S_{\tau_{n}} I_{\left\{\tau_{n}<\infty, S_{\tau_{n}} \leq 0\right\}} I_{\left\{Z_{\tau_{n}} \leq \lambda_{0} \widehat{Z}_{\tau_{n}}\right\}}\right] \\
& \geq \lambda_{0} \liminf _{n} E\left[\widehat{Z}_{\tau_{n}} S_{\tau_{n}} I_{\left\{\tau_{n}<\infty, S_{\tau_{n}} \leq 0\right\}} I_{\left\{Z_{\tau_{n}} \leq \lambda_{0} \widehat{Z}_{\tau_{n}}\right\}}\right]=0
\end{aligned}
$$

Therefore

$$
\liminf _{n} E\left[-Z_{\tau_{n}} S_{\tau_{n}} I_{\left\{\tau_{n}<\infty, S_{\tau_{n}} \leq 0\right\}} I_{\left\{Z_{\tau_{n}}>\lambda_{0} \widehat{Z}_{\tau_{n}}\right\}}\right]>0
$$


Set

$$
A_{n}=\left\{\tau_{n}<\infty, S_{\tau_{n}} \leq 0, Z_{\tau_{n}}>\lambda_{0} \widehat{Z}_{\tau_{n}}\right\}
$$

Clearly, $A_{n} \downarrow \emptyset$ and for all $n$ the stopping time $\tau_{n}$ coincides with $\tau_{n} \wedge T$ on $A_{n}$. Since $\frac{Z_{T}}{Z_{\tau_{n}}} \in \mathcal{M}_{1}\left(\tau_{n}\right)$

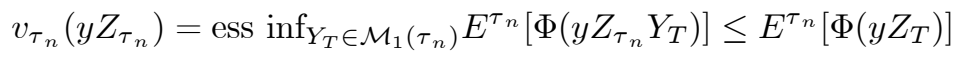

and we may compute

$$
\begin{aligned}
& E\left[\Phi\left(y Z_{T}\right) I_{A_{n}}\right]=E\left[E^{\tau_{n}}\left[\Phi\left(y Z_{T}\right)\right] I_{A_{n}}\right] \geq E\left[v_{\tau_{n}}\left(y Z_{\tau_{n}}\right) I_{A_{n}}\right] \\
\geq & c_{1} E\left[y Z_{\tau_{n}} v_{\tau_{n}}^{\prime}\left(y Z_{\tau_{n}}\right) I_{A_{n}}\right]-c_{2} E\left[\left(y Z_{\tau_{n}}+1\right) I_{A_{n}}\right]
\end{aligned}
$$

where we used Proposition 12 item 3) applied to the stopping time $\tau_{n} \wedge T$ and $c_{1}, c_{2}>0$ are suitable constants. Now, $E\left[\left(y Z_{\tau_{n}}+1\right) I_{A_{n}}\right]=y Q\left(A_{n}\right)+P\left(A_{n}\right) \rightarrow 0$ as $n \rightarrow \infty$ so that

$$
\liminf _{n} E\left[v_{\tau_{n}}\left(y Z_{\tau_{n}}\right) I_{A_{n}}\right] \geq \liminf _{n} c_{1} E\left[y Z_{\tau_{n}} v_{\tau_{n}}^{\prime}\left(y Z_{\tau_{n}}\right) I_{A_{n}}\right]
$$

By the monotonicity of $v_{\tau_{n}}^{\prime}$, proved in Proposition 12 item 4),

$$
E\left[y Z_{\tau_{n}} v_{\tau_{n}}^{\prime}\left(y Z_{\tau_{n}}\right) I_{A_{n}}\right] \geq E\left[y Z_{\tau_{n}} v_{\tau_{n}}^{\prime}\left(y \widehat{Z}_{\tau_{n}}\right) I_{A_{n}}\right]
$$

By the fundamental relation between primal and dual optima in the utility maximization (6), the primal optimal solution can be written as $x+\left(H_{x} \cdot X\right)_{T}=$ $-\Phi^{\prime}\left(y \widehat{Z}_{T}\right)$ (recall that $\left.\left(u^{\prime}\right)^{-1}=-\Phi^{\prime}\right)$ and the optimal wealth process $S=$ $x+H_{x} \cdot X$ is a $\widehat{Q}$-martingale. As a consequence, for any stopping time $\tau \leq T$

$$
S_{\tau}=E_{\widehat{Q}}^{\tau}\left[x+\left(H_{x} \cdot X\right)_{T}\right]=-E^{\tau}\left[\Phi^{\prime}\left(y \widehat{Z}_{T}\right) \frac{\widehat{Z}_{T}}{\widehat{Z}_{\tau}}\right]=-v_{\tau}^{\prime}\left(y \widehat{Z}_{\tau}\right)
$$

where the last equality follows from equation (22). Thus

$$
E\left[y Z_{\tau_{n}} v_{\tau_{n}}^{\prime}\left(y \widehat{Z}_{\tau_{n}}\right) I_{A_{n}}\right]=E\left[-y Z_{\tau_{n}} S_{\tau_{n}} I_{A_{n}}\right]
$$

From (26), (27), (28), (29), and (25) we finally obtain

$$
\liminf _{n} E\left[\Phi\left(y Z_{T}\right) I_{A_{n}}\right] \geq \liminf _{n} c_{1} E\left[-y Z_{\tau_{n}} S_{\tau_{n}} I_{A_{n}}\right]>0
$$

and this contradicts the fact that $Q$ has finite entropy, since $A_{n} \downarrow \emptyset$.

\subsection{Proof of of Item (b)}

When $u$ is exponential, thanks to the stability by concatenation of $\mathcal{M}$ (Lemma 7) and to the fact that $\left(H_{x} \cdot X\right)_{T} \in L^{1}(Q)$ for all $Q \in \mathcal{M} \cap \mathcal{P}_{\Phi}$, one can repeat word by word the proof in [KSO2] to show that the true martingale property of the optimal wealth process is preserved. 


\section{References}

[AS94] J.P. Ansel and C. Stricker. Couverture des Actifs contingents et prix maximum. Ann. Inst. H. Poincaré 30, 303-315, 1994.

[BF05] S. Biagini and M. Frittelli. Utility Maximization in Incomplete Markets for Unbounded Processes. Fin. \& Stoch. 9, 493-517, 2005.

[Del06] F. Delbaen. The structure of m-stable sets and of the set of risk neutral measures. Sem. Prob. XXXIX, 2006.

[DGR+02] F. Delbaen, P. Grandits, T. Rheinlaender, D. Samperi, M. Schweizer, and C. Stricker. Exponential Hedging and Entropic Penalties. Math. Fin. 12/2, 99-123, 2002.

[DS98] F. Delbaen and W. Schachermayer. The Fundamental Theorem of Asset Pricing for Unbounded Stochastic Processes. Math. Ann. 312, 215-250, 1998.

[DS99] F. Delbaen and W. Schachermayer. A Compactness Principle for Bounded Sequences of Martingales with Applications. Proc. of the Seminar on Stoch. Analysis, Random Fields and Appl., Progr. in Prob. 45, 137-173, 1999.

[HP81] J.M. Harrison and S.R. Pliska. Martingales and stochastic integrals in the theory of continuous trading. Stoch. Proc. \& Appl. 11, 215260, 1981.

[KS02] Y. Kabanov and C. Stricker. On the Optimal Portfolio for the Exponential Utility Maximization: Remarks to the Six-Authors Paper. Math. Fin. 12/2, 125-134, 2002.

[Pli86] S.R. Pliska. A stochastic calculus model of continuous trading. Math. Oper. Res. 11/2 371-382, 1986.

[Sch94] W. Schachermayer. Martingale Measures for discrete time processes with infinite horizon. Math. Fin. 4, 25-55, 1994.

[Sch01] W. Schachermayer. Optimal investment in incomplete markets when wealth may become negative. Ann. Appl. Prob. 11/3, 694-734, 2001.

[Sch03] W. Schachermayer. A super-martingale property of the optimal portfolio process. Fin. \& Stoch. 4, 433-457, 2003. 